\title{
Measurement of the Charge-Averaged Elastic Lepton-Proton Scattering Cross Section by the OLYMPUS Experiment
}

J. C. Bernauer $\odot,{ }^{1, \dagger}$ A. Schmidt $\odot,{ }^{1, *}$ B. S. Henderson, ${ }^{1}$ L. D. Ice, ${ }^{2}$ D. Khaneft, ${ }^{3}$ C. O'Connor, ${ }^{1}$ R. Russell, ${ }^{1}$ N. Akopov, ${ }^{4}$ R. Alarcon, ${ }^{2}$ O. Ates, ${ }^{5}$ A. Avetisyan, ${ }^{4}$ R. Beck,${ }^{6}$ S. Belostotski, ${ }^{7,}$ J. Bessuille, ${ }^{1}$ F. Brinker, ${ }^{8}$ J. R. Calarco, ${ }^{9}$ V. Carassiti, ${ }^{10}$ E. Cisbani, ${ }^{11}$ G. Ciullo, ${ }^{10}$ M. Contalbrigo, ${ }^{10}$ R. De Leo, ${ }^{12}$ J. Diefenbach,${ }^{5}$ T. W. Donnelly, ${ }^{1}$ K. Dow, ${ }^{1}$ G. Elbakian, ${ }^{4}$ P. D. Eversheim, ${ }^{6}$ S. Frullani, ${ }^{11, *}$ Ch. Funke, ${ }^{6}$ G. Gavrilov, ${ }^{7}$ B. Gläser, ${ }^{3}$ N. Görrissen, ${ }^{8}$ D. K. Hasell, ${ }^{1}$ J. Hauschildt, ${ }^{8}$ Ph. Hoffmeister, ${ }^{6}$ Y. Holler, ${ }^{8}$ E. Ihloff, ${ }^{1}$ A. Izotov, ${ }^{7}$ R. Kaiser, ${ }^{13}$ G. Karyan, ${ }^{4}$ J. Kelsey, ${ }^{1}$ A. Kiselev, ${ }^{7}$ P. Klassen, ${ }^{6}$ A. Krivshich, ${ }^{7}$ M. Kohl ${ }^{5}$ I. Lehmann, ${ }^{13}$ P. Lenisa, ${ }^{10}$ D. Lenz, ${ }^{8}$ S. Lumsden, ${ }^{13,}{ }^{*}$ Y. Ma, ${ }^{3}$ F. Maas, ${ }^{3}$ H. Marukyan, ${ }^{4}$ O. Miklukho, ${ }^{7}$ R. G. Milner, ${ }^{1}$ A. Movsisyan, ${ }^{4,10}$ M. Murray, ${ }^{13}$ Y. Naryshkin, ${ }^{7}$ R. Perez Benito, ${ }^{3}$ R. Perrino, ${ }^{12}$ R. P. Redwine, ${ }^{1}$ D. Rodríguez Piñeiro, ${ }^{3}$ G. Rosner, ${ }^{13}$ U. Schneekloth, ${ }^{8}$ B. Seitz, ${ }^{13}$ M. Statera, ${ }^{10}$ A. Thiel, ${ }^{6}$ H. Vardanyan, ${ }^{4}$ D. Veretennikov, ${ }^{7}$ C. Vidal, ${ }^{1}$ A. Winnebeck, ${ }^{1}$ and V. Yeganov ${ }^{4}$

(OLYMPUS Collaboration)

\author{
${ }^{1}$ Massachusetts Institute of Technology, Cambridge, Massachusetts 02139, USA \\ ${ }^{2}$ Arizona State University, Tempe, Arizona 85287, USA \\ ${ }^{3}$ Johannes Gutenberg-Universität, Mainz, Germany \\ ${ }^{4}$ Alikhanyan National Science Laboratory (Yerevan Physics Institute), Yerevan, Armenia \\ ${ }^{5}$ Hampton University, Hampton, Virginia 23668, USA \\ ${ }^{6}$ Rheinische Friedrich-Wilhelms-Universität, Bonn, Germany \\ ${ }^{7}$ Petersburg Nuclear Physics Institute, Gatchina, Russia \\ ${ }^{8}$ Deutsches Elektronen-Synchrotron, Hamburg, Germany \\ ${ }^{9}$ University of New Hampshire, Durham, New Hampshire 03824, USA \\ ${ }^{10}$ Università degli Studi di Ferrara and Istituto Nazionale di Fisica Nucleare sezione di Ferrara, Ferrara, Italy \\ ${ }^{11}$ Istituto Nazionale di Fisica Nucleare sezione di Roma and Istituto Superiore di Sanità, Rome, Italy \\ ${ }^{12}$ Istituto Nazionale di Fisica Nucleare sezione di Bari, Bari, Italy \\ ${ }^{13}$ University of Glasgow, Glasgow, United Kingdom
}

(Received 13 August 2020; revised 17 March 2021; accepted 23 March 2021; published 21 April 2021)

We report the first measurement of the average of the electron-proton and positron-proton elastic scattering cross sections. This lepton charge-averaged cross section is insensitive to the leading effects of hard two-photon exchange, giving more robust access to the proton's electromagnetic form factors. The cross section was extracted from data taken by the OLYMPUS experiment at DESY, in which alternating stored electron and positron beams were scattered from a windowless gaseous hydrogen target. Elastic scattering events were identified from the coincident detection of the scattered lepton and recoil proton in a large-acceptance toroidal spectrometer. The luminosity was determined from the rates of Møller, Bhabha, and elastic scattering in forward electromagnetic calorimeters. The data provide some selectivity between existing form factor global fits and will provide valuable constraints to future fits.

DOI: 10.1103/PhysRevLett.126.162501

As the lightest stable composite particle emerging from quantum chromodynamics, the proton is one of the best testing grounds for our understanding of the strong force. One of the ways of characterizing the proton's internal quark-gluon structure is through measurements of elastic electron-proton scattering, from which the proton's electromagnetic form factors, $G_{E}$ and $G_{M}$, can be extracted. These form factors reveal information about how electric charge and current are distributed within (though this relationship is far from simple, see Ref. [1]), and provide a touchstone for the verification of theoretical descriptions and computational approaches. For large $Q^{2}$, the progress in precision measurements is hampered by the unresolved discrepancy between measurements of the proton's elastic form factor ratio, $\mu_{p} G_{E}^{p} / G_{M}^{p}$, using polarization techniques [2-9], and those obtained using the traditional Rosenbluth technique in unpolarized cross section measurements [10-15].

One hypothesis for the cause of this discrepancy is a contribution to the cross section from hard two-photon exchange (TPE), which is not included in standard radiative corrections and would affect the two measurement techniques differently [16-21]. 
Standard radiative correction prescriptions account for two-photon exchange only in the soft limit, in which one photon carries negligible momentum $[22,23]$. There is no model-independent formalism for calculating hard TPE. Some model-dependent calculations suggest that TPE is responsible for the form factor discrepancy [18-21] while others contradict that finding $[24,25]$. The current status of the recent experimental and theoretical progress on twophoton exchange is summarized in Ref. [26].

While most models predict negligible effects of hard two-photon exchange on measurements using polarization, such measurements can only extract the form factor ratio. A separation of $G_{E}$ and $G_{M}$ requires absolute measurements of the lepton-proton cross sections, which are affected by hard TPE. To leading order, TPE effects depend on the charge sign of the lepton. Therefore, a charge-averaged cross section is far less sensitive to TPE. We report here on the first precision determination of a charge-averaged cross section of $e^{ \pm}-p$ scattering.

OLYMPUS's main goal was to measure the ratio of the cross sections for positron-proton and electron-proton scattering, a quantity which gives direct access to the two-photon exchange correction. OLYMPUS was optimized for this purpose, and the results were published in Ref. [27]. However, careful further analysis allowed us to extract charge-averaged cross sections. They cover an interesting kinematical region, where existing form factor fits show a turn-over behavior for $G_{M}$, and where the existing data for $e^{-}-p$ scattering are somewhat lacking, leading to large model uncertainties.

Only a brief overview of the OLYMPUS experiment is given here, and we refer to Ref. [28] for a detailed description of the detector. OLYMPUS was the last experiment to take data at the DORIS electron-positron storage ring at DESY, Hamburg, Germany. In total, an integrated luminosity of $4.5 \mathrm{fb}^{-1}$ was collected. The $2.01 \mathrm{GeV}$ stored beams with up to $65 \mathrm{~mA}$ of current passed through an internal, unpolarized hydrogen gas target with an areal density of approximately $3 \times 10^{15}$ atoms $/ \mathrm{cm}^{2}$ [29]. The accelerator magnet power supplies were modified to allow the daily change of beam species.

The main detector, a toroidal magnetic spectrometer, was based on the former MIT-Bates BLAST detector [30], with the two horizontal sections instrumented with large acceptance $\left(20^{\circ}<\theta<80^{\circ},-15^{\circ}<\phi<15^{\circ}\right)$ drift chambers (DC) for 3D particle tracking and walls of time-of-flight scintillator bars (TOF) for triggering and particle identification. The data presented here were collected entirely with positive-tracksoutbending toroid polarity in order to suppress background rates in the DC, so that low-energy electrons were bent back to the beam axis and away from the detectors.

Two new detector systems were designed and built to monitor the luminosity. These were symmetric MøllerBhabha calorimeters (SYMB) at $1.29^{\circ}[31]$ and two telescopes of three triple gas electron multiplier (GEM) detectors [32] interleaved with three multiwire proportional chambers (MWPC) mounted at $12^{\circ}$.

The trigger system selected candidate events that resulted from a lepton and proton detected in coincidence in opposite sectors. The data were acquired and stored via the CBELSA/TAPS data acquisition system [33].

The positions of all detector elements were determined via optical surveys and the magnetic field was mapped throughout the complete tracking volume with the magnet unmoved from its final position in the experiment [34].

Acceptances, radiative corrections, and efficiencies were accounted for via a realistic Monte Carlo (MC) simulation. The MC parameters, for example, beam position and beam current, were adjusted dynamically to match the values recorded by the slow control system to simulate the time dependence of these quantities. This approach also rigorously captured the possible correlation between parameters. The MC simulation used a radiative event generator developed specifically for OLYMPUS [35,36]. This generator produced lepton-proton events weighted by several different radiative cross section models. In this Letter, we present the results following the Maximon-Tjon [23] prescription. Higher order radiative corrections are taken into account through exponentiation.

Particle trajectories and energy losses were simulated using GEANT4, with custom digitization routines to produce output identical in format to actual measured data. This step included efficiency and resolution simulations whose parameters were determined from data. Both the simulated and the real data were then analyzed with identical software.

Track reconstruction used a fast hierarchical pattern matching algorithm to identify track candidates. Initial track parameters were then determined via two distinct track fit algorithms. The design of the drift chambers and the running conditions in OLYMPUS led to some trackfitting ambiguities that were difficult for the algorithms to resolve. While the algorithms did well for most constellations, they failed for certain pathological cases. However, the two algorithms struggled in different cases, so that the combination of both algorithms secured the reconstruction with high efficiency over the whole phase space.

Particle identification was achieved by a combination of track curvature direction, indicating the particle charge, and the correlation between momentum and TOF to cleanly separate positrons from protons.

The efficiency of the drift chambers was determined by performing track reconstruction without considering one of the drift chamber superlayers and then considering whether or not hits were present in the ignored superlayer. This technique was used to develop highly granular efficiency maps of each drift cell. These maps were used directly in the detector simulation. While the majority of the drift cells had efficiency $>95 \%$, several had reduced efficiency, likely because of high discriminator thresholds. These 
inefficient cells had only a small effect on the overall tracking efficiency because of the redundancy of the six superlayers.

The efficiency of the time-of-flight scintillators was assessed using the combination of cosmic ray studies, data taken with a prescaled efficiency trigger, and GEANT4 simulation. The efficiency was greater than $99 \%$ for protons and greater than $97 \%$ for electrons and positrons. The TOF efficiency model was also implemented in the OLYMPUS simulation.

The track reconstruction efficiency was assessed by selecting elastically recoiling protons in one sector and looking for the corresponding scattered lepton in the other sector. Within the precision of the study $(\approx 1 \%)$, there was no indication of inefficiency beyond that caused by TOF and drift chamber inefficiencies, and we therefore assign a $1 \%$ normalization uncertainty for any tracking inefficiency. An additional 2\% absolute normalization was estimated for other sources not tested by this method, e.g., for the trigger efficiency.

Four independent elastic event selection routines were developed [35-38], which allowed us to assess the degree of event-selection bias. While the four approaches differ in detail, they all exploit the fact that, for a coincidence measurement of elastic scattering, the kinematics are overdetermined and that selection cuts on the self-consistency of the kinematics can be used to suppress inelastic background. The routine of Ref. [37] used wide cuts in lepton-proton vertex time correlation, vertex-position correlation, polarangle correlation, and momentum correlation to reduce inelastic background, before estimating any remaining background using sidebands in the azimuthal distribution of track pairs. The routine of Ref. [36] performed different selection cuts and is distinguished by performing particle-ID at the level of track pairs, rather than individual tracks. The routine of Ref. [35] examined background over a twodimensional space of polar and azimuthal angle correlation. The routine of Ref. [38] built an elastic-pair probability for track pairs based on their vertex, time, and angle correlations, and the missing energy assuming elastic kinematics. Low probability combinations were rejected. The surviving best pair for each event was then used for the rate extraction, with a background estimate based on the coplanarity in $Q^{2}$ slices.

The background remaining after elastic event selection was subtracted. The four analyses found similar levels of background for both lepton species and found that the background level was higher with increasing $Q^{2}$. Because their event selection cuts differed in tightness, the four analyses varied in the amount of background they subtracted, ranging between 5\% and 20\% for the highest $Q^{2}$ bin. Figure 1 shows an example of the background fit in one analysis for one of the highest $Q^{2}$ bins.

The total recorded data were screened for optimal running conditions, and a subset corresponding to

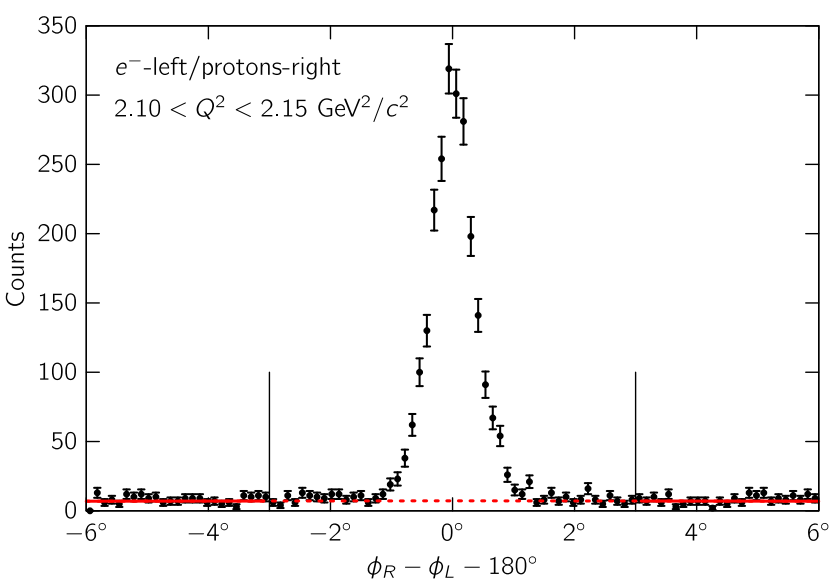

FIG. 1. Background was estimated and subtracted in Ref. [36] using fits to the sidebands of the distribution of the difference in azimuth of lepton $\left(\phi_{L}\right)$ and proton $\left(\phi_{R}\right)$ track pairs after all other elastic event selection criteria were applied. The background was largest at high $Q^{2}$, as shown here, with little difference between $e^{-}$and $e^{+}$modes.

$3.1 \mathrm{fb}^{-1}$ of integrated luminosity (the same subset as in Ref. [27]) was selected for the results presented here.

OLYMPUS was optimized for a measurement of the cross section ratio between the two beam species, and therefore it employed three independent systems to determine relative luminosity: from the elastic rate in the two $12^{\circ}$ telescopes, the Møller-Bhabha rate in the SYMB, and from the beam current and target density recorded by the slow control system. For an absolute measurement of the luminosity, none of the systems is optimal: (i) Fundamentally, the $12^{\circ}$ telescopes measure the same process as the main spectrometer and can therefore not give an absolute measurement. They could, however, extend the $Q^{2}$ range of the measurement so that an external determination of the cross section at this smaller value (for example, from a global fit), could establish the normalization and thus a quasiabsolute cross section for the remaining data points. However, the $12^{\circ}$ telescope acceptance and absolute efficiency are not known well enough to produce a sensible result. (ii) The slow control system could, in principle, give an absolute normalization. However, uncertainties from the target temperature, which affects the density, as well as the absolute calibration of the beam current could not be quantified with a reliable error estimate. (iii) The most robust SYMB analysis made use of multi-interaction events, in which a symmetric Møller or Bhabha event occurred in the same bunch as an unrelated forward-scattering elastic $e p$ event. This method takes advantage of the cancellation of many systematic effects when determining the relative luminosity between beam species. However, these effects do not cancel in the determination of the absolute luminosity, resulting in an uncertainty of $7 \%$. This method is used for normalizing the cross sections reported in this work. We note that the results of the SYMB and slow control differ only by about $1 \%$. 
TABLE I. Contributions to the systematic uncertainty in the global normalization.

\begin{tabular}{lc}
\hline \hline Source & Uncertainty in the normalization \\
\hline Luminosity & $7.0 \%$ \\
Efficiency & $2.0 \%$ \\
Event selection & $1.5 \%$ \\
Track reconstruction & $1.0 \%$ \\
Detector acceptance & $0.7 \%$ \\
Live-time correction & $0.5 \%$ \\
Total & $7.5 \%$ \\
\hline \hline
\end{tabular}

We report the cross section determined from the average of the results of the four independent analyses. We further use the variance between the analyses to estimate systematic uncertainties from event selection choices. However, we first remove the effect of normalization differences between the analyses. We find, for each analysis, the normalization factor that minimizes the difference of the analysis to the original average. After renormalization, we then determine the remaining variance and use this as an additional point-to-point uncertainty. The standard deviation of the normalization constants, $1.5 \%$, is added as an additional contribution to the global normalization uncertainty. The systematic difference between cross sections determined from the lepton-left-proton-right versus proton-right-lepton-left topologies is used to assess the systematic uncertainty from mis-modeling of the detector acceptance $(0.7 \%)$. In total, we achieve a global normalization uncertainty of $7.5 \%$, dominated by the luminosity uncertainty. Table I gives an overview.
The OLYMPUS determination of the charge-average cross section, as a function of $\epsilon$ and $Q^{2}$ is provided in Table II. A comparison of our results with a selection of fits is shown in Fig. 2. The fits presented here use different methods to minimize the influence of TPE on the extracted form factors. All use both Rosenbluth as well as polarized data in their fits, and assume that the influence of TPE on the ratio extracted from polarized data is minimal. Kelly [39] omits $G_{E}$ results for $Q^{2}>1(\mathrm{GeV} / c)^{2}$ and relies on ratio determinations from polarized experiments and $G_{M}$ values extracted from $e^{-}-p$ scattering, but does not correct them for hard TPE effects. While the effect of TPE on the extraction is small compared to the effect on $G_{E}$ at these $Q^{2}$, it is not clear a priori how large the effect is, and how the uncorrected data at smaller $Q^{2}$ affect the high$Q^{2}$ behavior. Arrington [40] uses a phenomenological correction to the cross sections with a linear dependence in $\epsilon$ and fixed scale of 6\%. Arrington et al. [41] and Ye et al. [42] use theoretical TPE calculations and complement them for data points $>1(\mathrm{GeV} / c)^{2}$ with an ad hoc additional effect that is linear in $\epsilon$ and scaled with logarithmic dependence on $Q^{2}$. Bernauer [29] uses a two-parameter phenomenological model, a combination of the Feshbach correction, valid at $Q^{2}=0$, and a linear model with logarithmic scaling in $Q^{2}$, applied to data at all $Q^{2}$, fitting form factor parameters and TPE parameters together.

The data presented here connect the well-constrained region below $1(\mathrm{GeV} / c)^{2}$ with the region between 1 and $2(\mathrm{GeV} / c)^{2}$ where TPE effects are more prominent. The fit by Bernauer prefers a strong cusp-like structure in $G_{M}$ around $1.3(\mathrm{GeV} / \mathrm{c})^{2}$, while the other, less flexible, fits,

TABLE II. Cross sections measured by OLYMPUS, using the exponentiated Maximon and Tjon radiative corrections prescription. Uncertainties are statistical and point-to-point systematic. There is a further $7.5 \%$ normalization uncertainty that is common to all data points.

\begin{tabular}{lcccc}
\hline \hline$\left\langle Q^{2}\right\rangle\left[\mathrm{GeV}^{2} / c^{2}\right]$ & $\langle\epsilon\rangle$ & $\sigma_{e^{-} p} /$ std dipole & $\sigma_{e^{+} p} /$ std dipole & Avg. $\sigma_{e p} /$ std dipole \\
\hline 0.624 & 0.898 & $1.0140 \pm 0.0014 \pm 0.0039$ & $1.0097 \pm 0.0013 \pm 0.0031$ & $1.0119 \pm 0.0010 \pm 0.0027$ \\
0.674 & 0.887 & $1.0155 \pm 0.0015 \pm 0.0043$ & $1.0076 \pm 0.0015 \pm 0.0037$ & $1.0116 \pm 0.0011 \pm 0.0028$ \\
0.724 & 0.876 & $1.0236 \pm 0.0017 \pm 0.0019$ & $1.0169 \pm 0.0016 \pm 0.0053$ & $1.0202 \pm 0.0012 \pm 0.0030$ \\
0.774 & 0.865 & $1.0361 \pm 0.0019 \pm 0.0024$ & $1.0287 \pm 0.0019 \pm 0.0062$ & $1.0324 \pm 0.0013 \pm 0.0039$ \\
0.824 & 0.853 & $1.0475 \pm 0.0022 \pm 0.0048$ & $1.0397 \pm 0.0021 \pm 0.0069$ & $1.0436 \pm 0.0015 \pm 0.0053$ \\
0.874 & 0.841 & $1.0496 \pm 0.0024 \pm 0.0060$ & $1.0451 \pm 0.0023 \pm 0.0025$ & $1.0473 \pm 0.0017 \pm 0.0039$ \\
0.924 & 0.829 & $1.0473 \pm 0.0028 \pm 0.0060$ & $1.0443 \pm 0.0027 \pm 0.0039$ & $1.0458 \pm 0.0019 \pm 0.0045$ \\
0.974 & 0.816 & $1.0545 \pm 0.0031 \pm 0.0043$ & $1.0547 \pm 0.0029 \pm 0.0061$ & $1.0546 \pm 0.0021 \pm 0.0051$ \\
1.024 & 0.803 & $1.0622 \pm 0.0034 \pm 0.0055$ & $1.0591 \pm 0.0033 \pm 0.0043$ & $1.0606 \pm 0.0024 \pm 0.0042$ \\
1.074 & 0.789 & $1.0600 \pm 0.0038 \pm 0.0082$ & $1.0553 \pm 0.0036 \pm 0.0034$ & $1.0576 \pm 0.0026 \pm 0.0040$ \\
1.124 & 0.775 & $1.0619 \pm 0.0042 \pm 0.0059$ & $1.0577 \pm 0.0040 \pm 0.0044$ & $1.0598 \pm 0.0029 \pm 0.0036$ \\
1.174 & 0.761 & $1.0653 \pm 0.0047 \pm 0.0064$ & $1.0663 \pm 0.0044 \pm 0.0056$ & $1.0658 \pm 0.0032 \pm 0.0049$ \\
1.246 & 0.739 & $1.0729 \pm 0.0038 \pm 0.0080$ & $1.0730 \pm 0.0036 \pm 0.0092$ & $1.0729 \pm 0.0026 \pm 0.0084$ \\
1.347 & 0.708 & $1.0769 \pm 0.0046 \pm 0.0059$ & $1.0743 \pm 0.0042 \pm 0.0045$ & $1.0756 \pm 0.0031 \pm 0.0043$ \\
1.447 & 0.676 & $1.0976 \pm 0.0054 \pm 0.0026$ & $1.0864 \pm 0.0049 \pm 0.0077$ & $1.0920 \pm 0.0036 \pm 0.0044$ \\
1.568 & 0.635 & $1.0944 \pm 0.0054 \pm 0.0054$ & $1.1058 \pm 0.0050 \pm 0.0065$ & $1.1001 \pm 0.0037 \pm 0.0049$ \\
1.718 & 0.581 & $1.1125 \pm 0.0066 \pm 0.0078$ & $1.1160 \pm 0.0061 \pm 0.0042$ & $1.1142 \pm 0.0045 \pm 0.0045$ \\
1.868 & 0.524 & $1.1325 \pm 0.0082 \pm 0.0117$ & $1.1338 \pm 0.0076 \pm 0.0052$ & $1.1331 \pm 0.0056 \pm 0.0070$ \\
2.038 & 0.456 & $1.1326 \pm 0.0090 \pm 0.0128$ & $1.1500 \pm 0.0084 \pm 0.0091$ & $1.1413 \pm 0.0061 \pm 0.0092$ \\
\hline \hline
\end{tabular}




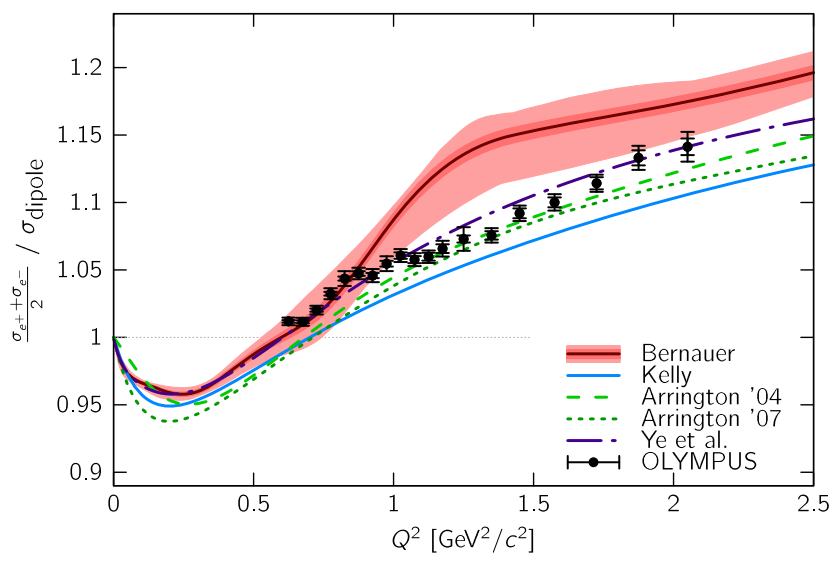

FIG. 2. The data for the charge-average cross section as a function of $Q^{2}$, in comparison with a series of predictions from form factor fits [39-43]. The Bernauer [43] prediction is shown with statistical (inner band) and model dependency systematical error (added linearly to statistical error, outer band). As can be deduced from the width of the bands and the differences between the models, prior data do not strongly constrain models in the range of $0.8<Q^{2}<2.5 \mathrm{GeV}^{2} / c^{2}$; this work can provide some remedy.

have a smoother transition. The data seem to be in better agreement with the latter, but a more detailed study of the effects of the new dataset on form factor fits must follow.

The advantage of the charge-averaging technique is that it suppresses all of the charge-odd radiative corrections. The suppression of hard TPE is advantageous because of the uncertainties associated with calculating it, but there may be additional benefits as well. Bremsstrahlung from the proton poses a similar challenge to hard TPE since it depends on an off-shell proton current. The interference term between electron and proton bremsstrahlung is one of the suppressed charge-odd effects, which, combined, grow in magnitude to become a sizable fraction of the total correction at higher $Q^{2}$, shown in Fig. 3. By forming the

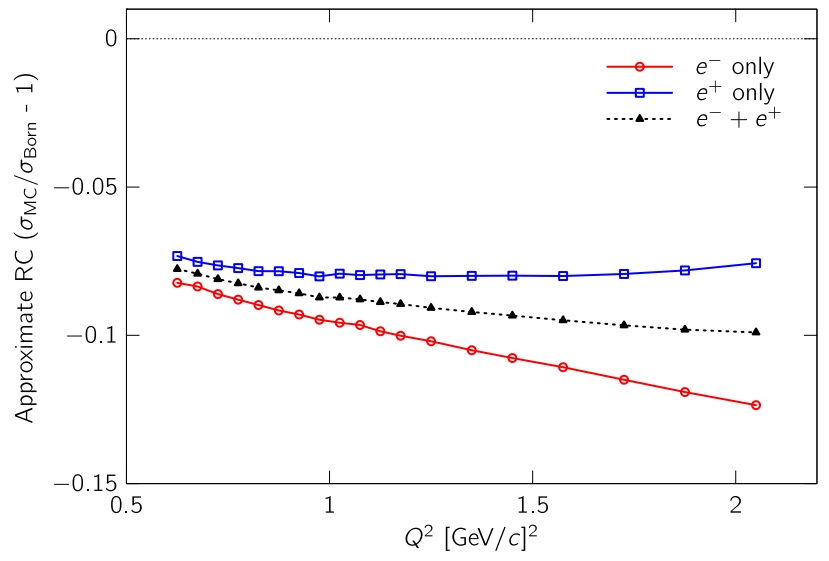

FIG. 3. The approximate radiative correction, estimated by taking the ratio of the simulated cross sections with and without the inclusion of radiative effects. The charge-odd contribution is a sizeable fraction of the total at high $Q^{2}$. charge average, the dominant part of the radiative correction is from radiation from the electron legs, which is under better theoretical control.

We thank the DORIS machine group and the various DESY groups that made this experiment possible. We gratefully acknowledge the numerous funding agencies: the Science Committee of Armenia, Grant No. 18T-1C180, the Deutsche Forschungsgemeinschaft, the European Community-Research Infrastructure Activity, the United Kingdom Science and Technology Facilities Council and the Scottish Universities Physics Alliance, the United States Department of Energy and the National Science Foundation, and the Ministry of Education and Science of the Russian Federation. R. G. M. also acknowledges the generous support of the Alexander von Humboldt Foundation, Germany. M. K. is partially supported by Jefferson Lab.

\footnotetext{
*Deceased.

jan.bernauer@stonybrook.edu

Present address: Stony Brook University, Stony Brook, New York 11794, USA and Riken BNL Research Center, Upton, New York 11793, USA.

\$axelschmidt@gwu.edu

Present address: George Washington University, Washington, DC 20052, USA.

[1] G. A. Miller, Defining the proton radius: A unified treatment, Phys. Rev. C 99, 035202 (2019).

[2] B. Hu et al., Polarization transfer in the ${ }^{2} \mathrm{H}\left(\vec{e}, e^{\prime} \vec{p}\right) n$ reaction up to $Q^{2}=1.61(\mathrm{GeV} / c)^{2}$, Phys. Rev. C 73, 064004 (2006).
}

[3] G. MacLachlan et al., The ratio of proton electromagnetic form factors via recoil polarimetry at $Q^{2}=1.13(\mathrm{GeV} / c)^{2}$, Nucl. Phys. A764, 261 (2006).

[4] O. Gayou et al., Measurements of the elastic electromagnetic form factor ratio $\mu_{p} G_{E_{p}} / G_{M_{p}}$ via polarization transfer, Phys. Rev. C 64, 038202 (2001).

[5] V. Punjabi et al., Proton elastic form factor ratios to $Q^{2}=3.5 \mathrm{GeV}^{2}$ by polarization transfer, Phys. Rev. C 71, 055202 (2005).

[6] M. K. Jones et al., Proton $G_{E} / G_{M}$ from beam-target asymmetry, Phys. Rev. C 74, 035201 (2006).

[7] A. J. R. Puckett et al., Recoil Polarization Measurements of the Proton Electromagnetic Form Factor Ratio to $Q^{2}=8.5 \mathrm{GeV}^{2}$, Phys. Rev. Lett. 104, 242301 (2010).

[8] M. Paolone et al., Polarization Transfer in the ${ }^{4} \mathrm{He}\left(\vec{e}, e^{\prime} \vec{p}\right)^{3} \mathrm{H}$ Reaction at $Q^{2}=0.8$ and $1.3(\mathrm{GeV} / c)^{2}$, Phys. Rev. Lett. 105, 072001 (2010).

[9] A. J. R. Puckett et al., Final analysis of proton form factor ratio data at $Q^{2}=4.0,4.8$, and $5.6 \mathrm{GeV}^{2}$, Phys. Rev. 85, 045203 (2012).

[10] J. Litt et al., Measurement of the ratio of the proton formfactors, $G_{E} / G_{M}$, at high momentum transfers and the question of scaling, Phys. Lett. 31B, 40 (1970).

[11] W. Bartel, F.-W. Büsser, W.-R. Dix, R. Felst, D. Harms, H. Krehbiel, P.E. Kuhlmann, J. McElroy, J. Meyer, and G. Weber, Measurement of proton and neutron electromagnetic 
form factors at squared four-momentum transfers up to $3(\mathrm{GeV} / c)^{2}$, Nucl. Phys. B58, 429 (1973).

[12] L. Andivahis et al., Measurements of the electric and magnetic form factors of the proton from $Q^{2}=1.75$ to $8.83 \sim(\mathrm{GeV} / c)^{2}$, Phys. Rev. D 50, 5491 (1994).

[13] R. C. Walker et al., Measurements of the proton elastic form factors for $1 \leq Q^{2} \leq 3(\mathrm{GeV} / c)^{2}$ at SLAC, Phys. Rev. D 49, 5671 (1994).

[14] M. E. Christy et al., Measurements of electron-proton elastic cross sections for $0.4<Q^{2}<5.5(\mathrm{GeV} / c)^{2}$, Phys. Rev. C 70, 015206 (2004).

[15] I. A. Qattan et al., Precision Rosenbluth Measurement of the Proton Elastic form Factors, Phys. Rev. Lett. 94, 142301 (2005).

[16] P. A. M. Guichon and M. Vanderhaeghen, How to Reconcile the Rosenbluth and the Polarization Transfer Method in the Measurement of the Proton Form Factors, Phys. Rev. Lett. 91, 142303 (2003).

[17] P. G. Blunden, W. Melnitchouk, and J. A. Tjon, Two Photon Exchange and Elastic Electron Proton Scattering, Phys. Rev. Lett. 91, 142304 (2003).

[18] Y. C. Chen, A. Afanasev, S. J. Brodsky, C. E. Carlson, and M. Vanderhaeghen, Partonic Calculation of the Two-Photon Exchange Contribution to Elastic Electron-Proton Scattering at Large Momentum Transfer, Phys. Rev. Lett. 93, 122301 (2004).

[19] A. V. Afanasev, S. J. Brodsky, C. E. Carlson, Y.-C. Chen, and M. Vanderhaeghen, Two-photon exchange contribution to elastic electron-nucleon scattering at large momentum transfer, Phys. Rev. D 72, 013008 (2005).

[20] P. G. Blunden, W. Melnitchouk, and J. A. Tjon, Two-photon exchange in elastic electron-nucleon scattering, Phys. Rev. C 72, 034612 (2005).

[21] S. Kondratyuk, P. G. Blunden, W. Melnitchouk, and J. A. Tjon, $\Delta$ Resonance Contribution to Two-Photon Exchange in Electron-Proton Scattering, Phys. Rev. Lett. 95, 172503 (2005).

[22] L. W. Mo and Y.-S. Tsai, Radiative Corrections to Elastic and Inelastic ep and $\mu p$ Scattering, Rev. Mod. Phys. 41, 205 (1969).

[23] L. C. Maximon and J. A. Tjon, Radiative corrections to electron proton scattering, Phys. Rev. C 62, 054320 (2000).

[24] Yu. M. Bystritskiy, E. A. Kuraev, and E. Tomasi-Gustafsson, Structure function method applied to polarized and unpolarized electron-proton scattering: A solution of the GE (p)/GM(p) discrepancy, Phys. Rev. C 75, 015207 (2007).

[25] E. A. Kuraev, V. V. Bytev, S. Bakmaev, and E. TomasiGustafsson, Charge asymmetry for electron (positron)proton elastic scattering at large angle, Phys. Rev. C 78, 015205 (2008).
[26] A. Afanasev, P. G. Blunden, D. Hasell, and B. A. Raue, Two-photon exchange in elastic electron-proton scattering, Prog. Part. Nucl. Phys. 95, 245 (2017).

[27] B. S. Henderson et al. (OLYMPUS Collaboration), Hard Two-Photon Contribution to Elastic Lepton-Proton Scattering: Determined by the OLYMPUS Experiment, Phys. Rev. Lett. 118, 092501 (2017).

[28] R. Milner, D. K. Hasell, M. Kohl, U. Schneekloth et al., The OLYMPUS Experiment, Nucl. Instrum. Methods Phys. Res., Sect. A 741, 1 (2014).

[29] J. C. Bernauer, V. Carassiti, G. Ciullo, B. S. Henderson, E. Ihloff, J. Kelsey, P. Lenisa, R. Milner, A. Schmidt, and M. Statera, The OLYMPUS Internal Hydrogen Target, Nucl. Instrum. Methods Phys. Res., Sect. A 755, 20 (2014).

[30] D. K. Hasell et al., The BLAST experiment, Nucl. Instrum. Methods Phys. Res., Sect. A 603, 247 (2009).

[31] R. P. Benito, D. Khaneft, C. O'Connor, L. Capozza, J. Diefenbach, B. Gläser, Y. Ma, F. E. Maas, and D. R. Piñeiro, Design and performance of a lead fluoride detector as a luminosity monitor, Nucl. Instrum. Methods Phys. Res., Sect. A 826, 6 (2016).

[32] O. Ates, Ph. D. thesis, Hampton University, Hampton, Virginia, 2014.

[33] A. Thiel et al., Well-Established Nucleon Resonances Revisited by Double-Polarization Measurements, Phys. Rev. Lett. 109, 102001 (2012).

[34] J. C. Bernauer et al., Measurement and tricubic interpolation of the magnetic field for the OLYMPUS experiment, Nucl. Instrum. Methods Phys. Res., Sect. A 823, 9 (2016).

[35] R. L. Russell, Ph. D. thesis, Massachusetts Institute of Technology, Cambridge, Massachusetts, 2016.

[36] A. Schmidt, Ph. D. thesis, Massachusetts Institute of Technology, Cambridge, Massachusetts, 2016.

[37] B. S. Henderson, Ph.D. thesis, Massachusetts Institute of Technology, Cambridge, Massachusetts, 2016.

[38] J. C. Bernauer, Elastic event selection (2016) (unpublished).

[39] J. J. Kelly, Simple parametrization of nucleon form factors, Phys. Rev. C 70, 068202 (2004).

[40] J. Arrington, Implications of the discrepancy between proton form-factor measurements, Phys. Rev. C 69, 022201(R) (2004).

[41] J. Arrington, W. Melnitchouk, and J.A. Tjon, Global analysis of proton elastic form factor data with two-photon exchange corrections, Phys. Rev. C 76, 035205 (2007).

[42] Z. Ye, J. Arrington, R. J. Hill, and G. Lee, Proton and neutron electromagnetic form factors and uncertainties, Phys. Lett. B 777, 8 (2018).

[43] J.C. Bernauer et al. (A1 Collaboration), Electric and magnetic form factors of the proton, Phys. Rev. C 90, 015206 (2014). 